\title{
Functional Nucleic Acids for Pathogenic Bacteria Detection
}

Dingran Chang, ${ }^{\dagger,+}$ Sandy Zakaria, ${ }^{\ddagger+}$ Sahar Esmaeili Samani, ${ }^{\S}$ Yangyang Chang, ${ }^{\#}$ Carlos D. M. Filipe, ${ }^{\S}$ Leyla Soleymani, ${ }^{\star, \|}$ John D. Brennan, ${ }^{\perp}$ Meng Liu, ${ }^{*}, \#$ and Yingfu Li ${ }^{*}, \dagger,+, \$, \perp$

'Department of Biochemistry and Biomedical Sciences, Michael G. DeGroote Institute of Infectious Disease Research, ${ }^{\ddagger}$ School of Biomedical Engineering, ${ }^{\S}$ Department of Chemical Engineering, "Department of Engineering Physics, ${ }^{\perp}$ Biointerfaces Institute and Department of Chemistry and Chemical Biology, McMaster University, 1280 Main Street West, Hamilton, ON, L8S 4K1 (Canada).

"School of Environmental Science and Technology, Key Laboratory of Industrial Ecology and Environmental Engineering (Ministry of Education), Dalian University of Technology, Dalian, 116024 (China).

${ }^{+}$D.C. and S.Z. contributed equally.

Correspondence to Yingfu Li (liying@mcmaster.ca) and Meng Liu (mliu@dlut.edu.cn) 
Table S1. Summary of the number of infections and deaths for common infectious pathogens

\begin{tabular}{|c|c|c|}
\hline Infectious pathogens & Number of infections & Number of deaths \\
\hline SARS-CoV-21 & 174 million (by June 9, 2021) & 3.75 million (by June 9, 2021) \\
\hline $\begin{array}{l}\text { Mycobacterium } \\
\text { tuberculosis }^{2}\end{array}$ & 10 million (2019) & 1.4 million (2019) \\
\hline Plasmodium parasites ${ }^{3}$ & 229 million (2019) & $409,000(2019)$ \\
\hline Clostridium difficile ${ }^{4}$ & $223,900(2019, \mathrm{US})$ & $12,800(2019, \mathrm{US})$ \\
\hline Legionella pneumophila 5 & $10,000(2018, \mathrm{US})$ & $10 \%$ risk of death \\
\hline Helicobacter pylori $^{6}$ & 1.24 million (annually, US) & 14,500 (annually, US) \\
\hline $\begin{array}{c}\text { Methicillin-resistant } \\
\text { Staphylococcus aureus }\end{array}$ & $323,700(2019, \mathrm{US})$ & $10,600(2019, \mathrm{US})$ \\
\hline $\begin{array}{c}\text { Pseudomonas } \\
\text { aeruginosa }^{4}\end{array}$ & $32,600(2019, \mathrm{US})$ & $2,700(2019, \mathrm{US})$ \\
\hline $\begin{array}{l}\text { Pathogens that cause } \\
\text { Diarrhoeal disease }\end{array}$ & $\begin{array}{c}1.7 \text { billion cases of childhood } \\
\text { annually }\end{array}$ & $\begin{array}{l}525,000 \text { children } \\
\text { annually }\end{array}$ \\
\hline Drug-resistant pathogens ${ }^{4}$ & $2,868,700(2019, \mathrm{US})$ & $35,900(2019, \mathrm{US})$ \\
\hline
\end{tabular}


Table S2. RNA-cleaving fluorogenic DNAzymes (RFDs) isolated for various infectious bacteria

\begin{tabular}{|c|c|c|c|c|c|c|c|c|c|}
\hline \multirow[t]{2}{*}{ RFD Name } & \multirow{2}{*}{$\begin{array}{l}\text { Positive } \\
\text { Selection } \\
\text { Bacteria }\end{array}$} & \multirow{2}{*}{$\begin{array}{c}\text { Counter } \\
\text { Selection Bacteria }\end{array}$} & \multirow{2}{*}{\multicolumn{4}{|c|}{$\begin{array}{l}\text { Sequences }^{a} \\
\left(5^{\prime} \rightarrow 3^{\prime}\right)\end{array}$}} & \multicolumn{2}{|c|}{ Selectivity Test $^{\mathrm{b}}$} & \multirow[t]{2}{*}{ Activator } \\
\hline & & & & & & & Positive & Negative & \\
\hline RFD-EC1 $^{8}$ & $\overline{E . \text { coli }}$ & B. subtilis & $\begin{array}{l}\text { CACGG } \\
\text { TGTGC } \\
\text { GACCG } \\
\text { TGGGA } \\
\text { TTGTG } \\
\text { CTCTT } \\
\text { ATCAA }\end{array}$ & $\begin{array}{l}\text { ATCCI } \\
\text { GTTGI } \\
\text { GAACA } \\
\text { TGGAT } \\
\text { TGCAC } \\
\text { CCTAC } \\
\text { GA }\end{array}$ & $\begin{array}{l}\text { GACAA } \\
\text { CGAGA } \\
\text { CTACA } \\
\text { TTCTT } \\
\text { CTCCG } \\
\text { CFRQG }\end{array}$ & $\begin{array}{l}\text { GGATG } \\
\text { CCTGC } \\
\text { CTGTG } \\
\text { TACAG } \\
\text { TCCGA } \\
\text { GTTCG }\end{array}$ & $\overline{E . c}$ & $\begin{array}{c}\text { B.s, L.m, L.pl, } \\
\text { P.ac,A.o, P.p, B.d, } \\
\text { H.a, Y.r, O.g, A.x, } \\
\text { M.o, A.i, S.fo }\end{array}$ & $\begin{array}{l}\text { A } 30-50 \mathrm{kDa} \\
\text { protein }\end{array}$ \\
\hline RFD-CD19 & $\begin{array}{l}\text { C. difficile (the } \\
\text { strain of } \\
\text { BI/027-H) }\end{array}$ & $\begin{array}{c}\text { E. coli } \\
\text { B. subtilis } \\
\text { C. difficile CD630 }\end{array}$ & $\begin{array}{l}\text { CACGG } \\
\text { TGAGT } \\
\text { CGGAG } \\
\text { TCCGT } \\
\text { FRQGG }\end{array}$ & $\begin{array}{l}\text { ATCCI } \\
\text { GGATI } \\
\text { TCGGC } \\
\text { CCGAC } \\
\text { TTCGE }\end{array}$ & $\begin{array}{l}\text { GACAA } \\
\text { GGGGC } \\
\text { ACTAT } \\
\text { TCTTC } \\
\text { TCAAG }\end{array}$ & $\begin{array}{l}\text { GGATC } \\
\text { CTGCG } \\
\text { TCAGC } \\
\text { CTAGC } \\
\text { A }\end{array}$ & $\begin{array}{c}C . d \text { strain of BI/027- } \\
\mathrm{H}\end{array}$ & $\begin{array}{c}\text { C.d (other strains), } \\
\text { B,s, L.me, P.ac, P.p, } \\
\text { B.d, H.a, Y.r, O.g, } \\
\text { A.x, M.o, A.l, S.fo, } \\
\text { E.c }\end{array}$ & TcdC-24 \\
\hline DHp3T4 ${ }^{10}$ & H. pylori & $\begin{array}{l}\text { E. coli } \mathrm{O} 157: \mathrm{H} 7 \\
\text { C. difficile } \\
\text { S. typhimurium } \\
\text { B. subtilis } \\
\text { L. monocytogenes. }\end{array}$ & $\begin{array}{l}\text { CTATG } \\
\text { CACTA } \\
\text { TGGTC } \\
\text { CCGAG } \\
\text { TCG }\end{array}$ & $\begin{array}{l}\text { AACTC } \\
\text { CCAAC } \\
\text { TTTGC } \\
\text { GGTAC }\end{array}$ & $\begin{array}{l}\text { ACQRF } \\
\text { ATGCC } \\
\text { TATGT } \\
\text { AGCTC }\end{array}$ & $\begin{array}{l}\text { GACCT } \\
\text { ATCGA } \\
\text { GGGGT } \\
\text { TGAAC }\end{array}$ & H.P & $\begin{array}{c}\text { B.f, E.f, S.sa, L.m, S.t, } \\
\text { B.s, C.d, E.c, K.p. } \\
\text { F.n, P.a }\end{array}$ & $\begin{array}{c}\text { A } 50-100 \\
\text { kDa protein }\end{array}$ \\
\hline$\overline{\mathrm{LP}} 1^{11}$ & $\begin{array}{c}L . \\
\text { pneumophila }\end{array}$ & $\begin{array}{l}P . \text { aeruginosa } \\
\text { K. pneumonia }\end{array}$ & $\begin{array}{l}\text { CTATG } \\
\text { CACTA } \\
\text { CAATA } \\
\text { CAGCC } \\
\text { TAGAT } \\
\text { TCGGA }\end{array}$ & $\begin{array}{l}\text { AACTC } \\
\text { CCAAC } \\
\text { CCGAC } \\
\text { GATCZ } \\
\text { AAGCZ } \\
\text { GGCTI }\end{array}$ & $\begin{array}{l}\text { ACQRF } \\
\text { CAAGC } \\
\text { CCTTT } \\
\text { TACCT } \\
\text { CATCT } \\
\text { AG }\end{array}$ & $\begin{array}{l}\text { GACCT } \\
\text { ATGGA } \\
\text { CATTT } \\
\text { CAATG } \\
\text { TGTCA }\end{array}$ & L.P & $\begin{array}{l}\text { O.g, B.d, A.x, F.n, } \\
\text { S.s, E.f, L.m, B.s, V.p, } \\
\text { C.d, B.f, A.o, K.a, } \\
\text { K.p, E.a, E.cl, S.e, } \\
\text { E.c, S.s, S.f, Y.r, H.a, } \\
\text { S.fo, A.i, P.a }\end{array}$ & $\begin{array}{l}\text { A } 30-100 \\
\text { kDa protein }\end{array}$ \\
\hline RFA13-1 $^{12}$ & K. aerogenes & C. difficile & $\begin{array}{l}\text { CATCA } \\
\text { CACTA } \\
\text { TCCCT } \\
\text { TAAGA } \\
\text { CCAGC } \\
\text { TGATG }\end{array}$ & $\begin{array}{l}\text { GACTC } \\
\text { CCAAC } \\
\text { ACACC } \\
\text { TCTTI } \\
\text { GGCGC } \\
\text { TGGAZ }\end{array}$ & $\begin{array}{l}\text { CGQRF } \\
\text { CAACC } \\
\text { GGTAT } \\
\text { CAGGG } \\
\text { AGCGG } \\
\text { GCAGA }\end{array}$ & $\begin{array}{l}\text { AACCT } \\
\text { TAAAC } \\
\text { TTGGG } \\
\text { GGTGC } \\
\text { GAGGC } \\
\text { TGTCG }\end{array}$ & $\begin{array}{l}\text { K.a, K.p, E.cl, S.e } \\
\text { E.c, S.s, S.f, }\end{array}$ & $\begin{array}{l}\text { Y.r, H.a, S.fo, A.l, } \\
\text { L.p, O.g, B.d, A.x, } \\
\text { F.n, E.f, L.m,B.s, } \\
\text { C.d,A.O }\end{array}$ & $\begin{array}{c}\text { Activator is } \\
\text { RNase I from } \\
\text { Enterobacteri } \\
\text { aceae bacteria }\end{array}$ \\
\hline RFD-KP6 ${ }^{13}$ & K. pneumoniae & $\begin{array}{c}\text { K. pneumoniae, } E . \\
\text { coli, and } B . \\
\text { subtilis. }\end{array}$ & $\begin{array}{l}\text { CTATG } \\
\text { CACTA } \\
\text { ACCAA } \\
\text { ACTAA } \\
\text { GCTGC } \\
\text { TCTGA }\end{array}$ & $\begin{array}{l}\text { AACTC } \\
\text { CCAAC } \\
\text { CCATC } \\
\text { GAGAT } \\
\text { ACGTC } \\
\text { ACTCC }\end{array}$ & $\begin{array}{l}\text { ACQRF } \\
\text { ATGCC } \\
\text { ACTGG } \\
\text { TTCAG } \\
\text { GTAGG }\end{array}$ & $\begin{array}{l}\text { GACCT } \\
\text { ATCCT } \\
\text { TTTGT } \\
\text { GCATC } \\
\text { TGAGC }\end{array}$ & $\begin{array}{c}K . p, E . c(\text { weak } \\
\text { signal), B.c (weak } \\
\text { signal) }\end{array}$ & L.m, F.n & - \\
\hline
\end{tabular}

a) F: FAM-dT; Q: DABCYL-dT; R, adenine ribonucleotide

b) Abbreviation of bacteria: Acinetobacter lwoffii (A.l), Actinomyces orientalis (A.o), Achromobacter xylosoxidans (A.x), Brevundimonas diminuta (B.d), Bacterioied fagillis (B.f), Bacillus subtilis (B.s), Clostridium difficile (C.d), Enterobacter aerogenes (E.a), Enterobacter cloacae (E.cl), Escherichia coli (E.c), Enterococcus faecium (E.f), Fusobacterium nucleatum (F.n), Hafnia alvei (H.a), Klebsiella aerogenes (K.a), Klebsiella pneumoniae (K.p), Listeria monocytogenes (L.m), Leuconostoc mesenteroides(L.me), Legionella pneumophila(L.p), Lactobacillus planturum (L.pl), Moraxella osloensis (M.o), Ochrobactrum gringonese (O.g), Pseudomonas aeruginosa (P.a), Pediococcus acidilactici (P.ac), Pseudomonas peli (P.p), Salmonella enterica (S.e), Shigella flexneri (S.f), Serratia fonticola (S.fo), Shigella sonnei (S.s), Streptococcus salivarius (S.sa), Veillonella parvula (V.p), Vibrio parahemolyticus (V.pa), Yersinia ruckeri (Y.r). 
Table S3. Protein-binding DNA aptamers for Clostridium difficile detection

\begin{tabular}{|c|c|c|c|c|c|c|}
\hline \multirow{2}{*}{$\begin{array}{l}\text { Aptamer } \\
\text { Name }\end{array}$} & \multirow{2}{*}{$\begin{array}{c}\text { Protein } \\
\text { target }\end{array}$} & \multirow{2}{*}{\multicolumn{3}{|c|}{$\begin{array}{l}\text { Sequences } \\
\left(5^{\prime} \rightarrow 3^{\prime}\right)\end{array}$}} & \multicolumn{2}{|c|}{ Selectivity Test $^{\mathrm{a}}$} \\
\hline & & & & & Positive target & Negative target \\
\hline Capt- $2^{14}$ & $\begin{array}{l}\text { Recombinant glutamate } \\
\text { dehydrogenase (GDH) } \\
\text { from C.d }\end{array}$ & $\begin{array}{l}\text { GGAGG } \\
\text { CATCT }\end{array}$ & $\begin{array}{l}\text { TATTT AGTGC } \\
\text { CAAAC GACGT }\end{array}$ & $\begin{array}{l}\text { CAAGC } \\
\text { CTGAG }\end{array}$ & Recombinant GDH from $C . d$ & TcdA and TcdB from C.d \\
\hline Capt- $4^{14}$ & $\begin{array}{l}\text { Recombinant GDH } \\
\text { from } C . d\end{array}$ & $\begin{array}{l}\text { CGACC } \\
\text { CAATG }\end{array}$ & $\begin{array}{ll}\text { CGAAT } & \text { AGTGG } \\
\text { AGTGC } & \text { TTGTC }\end{array}$ & $\begin{array}{l}\text { CATAT } \\
\text { ATCTT }\end{array}$ & Recombinant GDH from $C . d$ & TcdA and TcdB from C.d \\
\hline Dapt $1^{15}$ & $\begin{array}{l}\text { Degraded native toxin } \\
\text { B from C.d }\end{array}$ & $\begin{array}{l}\text { AATAG } \\
\text { GGTGC } \\
\text { CTCTT }\end{array}$ & $\begin{array}{l}\text { TCTGG TTCGG } \\
\text { GATGT GTTAG } \\
\text { GTGTG }\end{array}$ & $\begin{array}{l}\text { TGCTT } \\
\text { TCAGA }\end{array}$ & $\begin{array}{l}\text { Degraded native toxin } \mathrm{B} \text {, fresh } \\
\text { native toxin } \mathrm{B} \text { and thermally } \\
\text { treated native toxin } \mathrm{B} \text { from } C . d\end{array}$ & $\begin{array}{c}\text { Recombinant toxin B, toxin A and } \\
\text { GDH from C.d }\end{array}$ \\
\hline ARH1t6 ${ }^{16}$ & RNase $\mathrm{H} 2$ from $C . d$ & $\begin{array}{l}\text { TTACG } \\
\text { GCCAG }\end{array}$ & 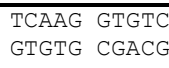 & $\begin{array}{l}\text { ACTCC } \\
\text { GTCGT }\end{array}$ & RNase $\mathrm{H} 2$ from $C . d$ & $\begin{array}{c}\text { RNase } \mathrm{H} 2 \text { from B.s, E.c, L.m, and } \\
\text { S.t }\end{array}$ \\
\hline
\end{tabular}

a) Abbreviation of bacteria: Bacillus subtilis (B.s), Clostridium difficile (C.d), Escherichia coli (E.c), Listeria monocytogenes (L.m), Salmonella Typhimurium (S.t). 
Table S4. Summary of FNA biosensors

\begin{tabular}{|c|c|c|c|c|c|}
\hline $\begin{array}{l}\text { FNAs biosensor } \\
\text { type }\end{array}$ & $\begin{array}{l}\text { Recognition } \\
\text { element }^{\mathbf{a}}\end{array}$ & Signal transducer & $\begin{array}{c}\text { Nucleic Acid } \\
\text { Amplification or target } \\
\text { enrichment }\end{array}$ & Sensitivity & Ref \\
\hline $\begin{array}{l}\text { Solution-based } \\
\text { fluorescent } \\
\text { biosensor }\end{array}$ & RFD-EC1 & $\begin{array}{c}\text { Separation of } \\
\text { fluorophore-quencher } \\
\text { pairs }\end{array}$ & Cell culturing (6 hrs) & Single cell of E. coli & 17 \\
\hline $\begin{array}{l}\text { Solution-based } \\
\text { fluorescent } \\
\text { biosensor }\end{array}$ & $\mathrm{EC} 1$ & $\begin{array}{l}\text { EvaGreen binding to } \\
\text { RCA products }\end{array}$ & $\begin{array}{c}\text { Rolling circle of } \\
\text { amplification (RCA) }\end{array}$ & 10 cells/mL of $E$. coli & 18 \\
\hline $\begin{array}{l}\text { Solution-based } \\
\text { fluorescent } \\
\text { biosensor }\end{array}$ & $\mathrm{EC} 1$ & $\begin{array}{l}\text { SYBR gold binding to } \\
\text { RCA products }\end{array}$ & RCA & 10 cells/mL E. coli & 19 \\
\hline $\begin{array}{l}\text { Solution-based } \\
\text { fluorescent } \\
\text { biosensor }\end{array}$ & $\mathrm{EC} 1$ & $\begin{array}{c}\text { G-quadruplex- } \\
\text { mediated fluorescence } \\
\text { enhancement }\end{array}$ & $\begin{array}{c}\text { Catalytic hairpin assembly } \\
\text { (CHA) }\end{array}$ & 50 cells/mL of $E$. coli & 20 \\
\hline $\begin{array}{l}\text { Paper-based } \\
\text { fluorescent } \\
\text { biosensor }\end{array}$ & EC1 & $\begin{array}{l}\text { Capture of fluorescent } \\
\text { DNA onto paper }\end{array}$ & $\begin{array}{c}\text { Surface-to-Surface product } \\
\text { enrichment strategy }\end{array}$ & 100 cells/mL of $E$. coli & 21 \\
\hline $\begin{array}{l}\text { Paper-based } \\
\text { fluorescent } \\
\text { biosensor }\end{array}$ & $\mathrm{EC} 1$ & $\begin{array}{l}\text { Capture of fluorescent } \\
\text { DNA onto paper }\end{array}$ & $\begin{array}{c}\text { No amplification or target } \\
\text { enrichment }\end{array}$ & 1000 cells/mL of $E$. coli & 22 \\
\hline $\begin{array}{l}\text { Paper-based } \\
\text { fluorescent } \\
\text { biosensor }\end{array}$ & RFD-EC1 & $\begin{array}{c}\text { Separation of } \\
\text { fluorophore-quencher } \\
\text { pairs }\end{array}$ & $\begin{array}{c}\text { No amplification or target } \\
\text { enrichment }\end{array}$ & 100 cells $/ \mathrm{mL}$ of $E$. coli & 23 \\
\hline $\begin{array}{l}\text { Paper-based } \\
\text { fluorescent } \\
\text { biosensor }\end{array}$ & GDH aptamer & $\begin{array}{l}\text { PW17 DNAzyme- } \\
\text { mediated color change }\end{array}$ & RCA & $\begin{array}{c}3 \mathrm{nM} \text { of glutamate } \\
\text { dehydrogenase }(\mathrm{GDH})\end{array}$ & 24 \\
\hline $\begin{array}{l}\text { Solution-based } \\
\text { colorimetric } \\
\text { biosensor }\end{array}$ & RFD-EC1 & $\begin{array}{l}\text { Urease-mediated } \\
\text { litmus color change }\end{array}$ & $\begin{array}{c}\text { No amplification or target } \\
\text { enrichment }\end{array}$ & 500 E. coli cells & 25 \\
\hline $\begin{array}{l}\text { Paper-based } \\
\text { colorimetric } \\
\text { biosensor }\end{array}$ & $\mathrm{EC} 1$ & $\begin{array}{l}\text { Urease-mediated } \\
\text { litmus color change }\end{array}$ & $\begin{array}{l}\text { Surface-to-Surface product } \\
\text { enrichment strategy }\end{array}$ & 100 cells $/ \mathrm{mL}$ of $E$. coli & 21 \\
\hline $\begin{array}{l}\text { Paper-based } \\
\text { colorimetric } \\
\text { biosensor }\end{array}$ & DHp3T4 & $\begin{array}{l}\text { Urease-mediated } \\
\text { litmus color change }\end{array}$ & $\begin{array}{c}\text { No amplification or target } \\
\text { enrichment }\end{array}$ & $\begin{array}{c}1000 \text { cells/mL of } H . \text { pylori } \\
\text { spiked into stool }\end{array}$ & 10 \\
\hline $\begin{array}{l}\text { Paper-based } \\
\text { colorimetric } \\
\text { biosensor }\end{array}$ & Dapt1T & $\begin{array}{c}\text { PW17 DNAzyme- } \\
\text { mediated color change }\end{array}$ & RCA & $60 \mathrm{pM}$ of toxin $\mathrm{B}$ & 15 \\
\hline $\begin{array}{l}\text { Electrochemical } \\
\text { biosensor }\end{array}$ & EC1 & $\begin{array}{l}\text { Methylene blue- } \\
\text { mediated } \\
\text { electrochemical signal }\end{array}$ & $\begin{array}{c}\text { No amplification or target } \\
\text { enrichment }\end{array}$ & $10 E$. coli cells in urine & 26 \\
\hline
\end{tabular}

a) EC1 is a simplified version of RFD-EC1 where the F and Q modifications are removed and the sequence is truncated and optimized. 


\section{References}

1. Template: COVID-19 Pandemic Data. In: Wikipedia. 2021. Accessed June 9, 2021. https://en.wikipedia.org/w/index.php?title=Template:COVID-

$19 \_$pandemic_data\&oldid $=1027759269$

2. Tuberculosis (TB). Accessed June 9, 2021. https://www.who.int/news-room/factsheets/detail/tuberculosis

3. Malaria. Accessed June 9, 2021. https://www.who.int/westernpacific/health-topics/malaria

4. CDC. Antibiotic-resistant Germs: New Threats. Centers for Disease Control and Prevention. Published March 2, 2021. Accessed May 27, 2021. https://www.cdc.gov/drugresistance/biggestthreats.html

5. Legionnaires Disease History, Burden, and Trends |CDC. Published March 25, 2021. Accessed June 9, 2021. https://www.cdc.gov/legionella/about/history.html

6. Institute of Medicine (US) Committee to Study Priorities for Vaccine Development; Stratton, K. R.; Durch, J. S.; Lawrence, R. S. Vaccines for the 21st Century: A Tool for Descisionmaking. Washington (DC): National Academies Press (US) 2000, Appendix 7, Helicobacter pylori.

7. Diarrhoeal Disease. Accessed June 9, 2021. https://www.who.int/news-room/factsheets/detail/diarrhoeal-disease

8. Ali, M. M.; Aguirre, S. D.; Lazim, H.; Li, Y. Fluorogenic DNAzyme Probes as Bacterial Indicators. Angew. Chem., Int. Ed. 2011, 123, 3835-3838.

9. Shen, Z.; Wu, Z.; Chang, D.; Zhang, W.; Tram, K.; Lee, C.; Kim, P.; Salena, B. J.; Li, Y. A Catalytic DNA Activated by a Specific Strain of Bacterial Pathogen. Angew. Chem., Int. Ed. 2016, 55, 24312434.

10. Ali, M. M.; Wolfe, M.; Tram, K.; Gu, J.; Filipe, C. D. M.; Li, Y; Brennan, J. D. A DNAzyme-Based Colorimetric Paper Sensor for Helicobacter pylori. Angew. Chem., Int. Ed. 2019, 58, 9907-9911.

11. Rothenbroker, M.; McConnell, E. M.; Gu, J.; Urbanus, L. M.; Samani, S. E.; Ensminger, A. W.; Filipe, C. D. M.; Li, Y. Selection and Characterization of an RNA-Cleaving DNAzyme Activated by Legionella pneumophila. Angew. Chem., Int. Ed. 2021, 60, 4782-4788.

12. Chang, D.; Chang, T.; Salena, B.; Li, Y. An Unintentional Discovery of a Fluorogenic DNA Probe for Ribonuclease I. ChemBioChem 2020, 21, 464-468.

13. Ali, M.M.; Slepenkin, A.; Peterson, E.; Zhao, W. A Simple DNAzyme-Based Fluorescent Assay for Klebsiella pneumoniae. ChemBioChem 2019, 20, 906-910.

14. Liu, M.; Yin, Q.; Chang, Y.; Zhang, Q.; Brennan, J. D.; Li, Y. In Vitro Selection of Circular DNA Aptamers for Biosensing Applications. Angew. Chem., Int. Ed. 2019, 58, 8013-8017. 
15. Liu, M.; Wang, J.; Chang, Y.; Zhang, Q.; Chang, D.; Hui, C. Y.; Brennan, J. D.; Li, Y. In Vitro Selection of a DNA Aptamer Targeting Degraded Protein Fragments for Biosensing. Angew. Chem., Int. Ed. 2020, 59, 7706-7710.

16. Li, J.; Gu, J.; Zhang, H.; Liu, R.; Zhang, W.; Mohammed-Elsabagh, M.; Xia, J.; Morrison, D.; Zakaria, S.; Chang, D.; Arrabi, A.; Li, Y. A Highly Specific DNA Aptamer for RNase H2 from Clostridium difficile. ACS Appl. Mater. Interfaces 2021, 13, 9464-9471.

17. Aguirre, S.; Ali, M.; Salena, B.; Li Y. A Sensitive DNA Enzyme-Based Fluorescent Assay for Bacterial Detection. Biomolecules 2013, 3, 563-577.

18. Liu, M.; Zhang, Q.; Li, Z.; Gu, J.; Brennan, J. D.; Li, Y. Programming a Topologically Constrained DNA Nanostructure into a Sensor. Nat Commun. 2016, 7, 12074.

19. Liu, M.; Zhang, Q.; Chang, D.; Gu, J.; Brennan, J. D.; Li, Y. A DNAzyme Feedback Amplification Strategy for Biosensing. Angew. Chem., Int. Ed. 2017, 56, 6142-6146.

20. Zhou, Z.; Brennan, J. D.; Li, Y. A Multi-component All-DNA Biosensing System Controlled by a DNAzyme. Angew. Chem., Int. Ed. 2020, 59, 10401-10405.

21. Samani, S. E.; Chang, D.; McConnell, E. M.; Rothenbroker, M.; Filipe, C. D. M.; Li, Y. Highly Sensitive RNA-cleaving DNAzyme Sensors from Surface-to-Surface Product Enrichment. ChemBioChem 2020, 21, 632-637.

22. Samani, S. E.; McConnell, E. M.; Chang, D.; Rothenbroker, M.; Filipe, C. D. M.; Li, Y. A SyringeBased DNAzyme Sensor for Bacterial Detection. Analysis \& Sensing 2021, 1, 95-100.

23. Ali, M. M.; Brown, C. L.; Jahanshahi-Anbuhi, S.; Kannan, B.; Li, Y.; Filipe, C. D. M.; Brennan, J. D. A Printed Multicomponent Paper Sensor for Bacterial Detection. Sci. Rep. 2017, 7, 12335.

24. Hui, C. Y.; Liu, M.; Li, Y.; Brennan, J. D. A Paper Sensor Printed with Multifunctional Bio/Nano Materials. Angew. Chem., Int. Ed. 2018, 57, 4549-4553.

25. Tram, K.; Kanda, P.; Salena, B. J.; Huan, S.; Li, Y. Translating Bacterial Detection by DNAzymes into a Litmus Test. Angew. Chem., Int. Ed. 2014, 126, 13013-13016.

26. Pandey, R.; Chang, D.; Smieja, M.; Hoare, T.; Li, Y.; Soleymani, L. Integrating Programmable RNAcleaving DNAzymes with Electrical Readout Enables Rapid and Culture-free Bacterial Detection using a Handheld Platform. Nat. Chem. 2021. doi: 10.1038/s41557-021-00718-x. 